\title{
Detecting Driving Awareness
}

\author{
Bruno Apolloni, Andrea Brega, Dario Malchiodi, and Cristian Mesiano \\ Dipartimento di Scienze dell'Informazione, Via Comelico 39/41, 20135 Milano, Italy \\ \{apolloni,brega,malchiodi,mesiano\}@dsi.unimi.it
}

\begin{abstract}
We consider the task of monitoring the awareness state of a driver engaged in attention demanding manoeuvres. A Boolean normal form launches a flag when the driver is paying special attention to his guiding. The contrasting analysis of these flags with the physical parameters of the car may alert a decision system whenever the driver awareness is judged unsatisfactory. The paper presents preliminary results showing the feasibility of the task.
\end{abstract}

\section{The Experiment}

We consider persons driving in a very good quality simulator that reproduces some typical phenomena in an interactive environment where they may cross or overpass other cars, follow a queue, face crossroads, etc. This simulator, which is in Queen University of Belfast, is equipped with a Biopac device having some not invasive sensors to record the electrochardiographic signals (ECG), the respiration (RSP), the galvanic skin response (GSR) and the skin temperature (SKT) of the subject at the sampling rate of $200 \mathrm{~Hz}$. A trial is constituted by a road trip that must be followed by the driver with different boundary conditions more or less affecting the driver emotional state. We sharply distinguish between unsoliciting conditions, by default, and soliciting conditions represented by periodically fast direction changes induced by cones in front of the car. The main cinematic parameters of the car, such as speed, acceleration, and wheel angle, are recorded during the trial.

\section{A Procedure from Sensory Data to Rules}

We may figure the whole procedure hosted by a hybrid multilayer perceptron where first layers compute subsymbolic functions like a conventional neural network, while the latter are arrays of Boolean gates computing formulas of increasing complexity. From an informational perspective the signal flows left to right evolving through representations increasingly compressed where the information is distributed in both the states of the processing elements and their connections. The training of this machinery is reported elsewhere [4]; here we will rather discuss the emerging features.

Since SKT is a constant due to simulation shortness, we extract ten features just from the first three signals, that we avoided to filter as this operation generally destroys relevant informations. We also didn't manage for removing motion artifacts since the features we will consider are insensitive to them. Eight features are conventional, consisting in distances between relevant points of the signals according to medical knowledge [5] (as sketched in Figure 1), while the other two are power spectrum and neural prediction shift. The power spectrum captures global properties of the 
ECG pulse and is computed within every pulse through usual Fast Fourier Transform processing. Finally a "neural" distance is computed between the actual ECG trajectory and the one 10 steps ahead forecasted by a specially featured recurrent neural network. This network is fed by (last line in Figure 1(b)) the current values of the three physiological signals and is embedded with both conventional sigmoidally activated neurons and symbolic ones expressly computing second and fifth order dynamics as baselines of the RSP and ECG signals respectively.

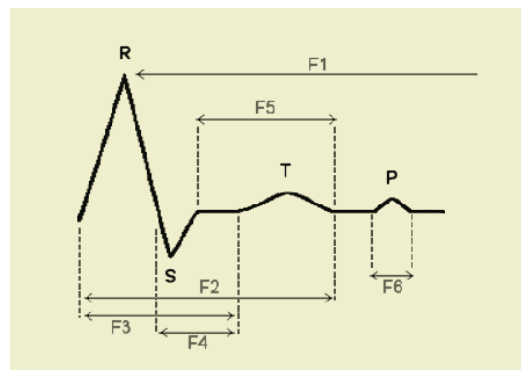

(a)

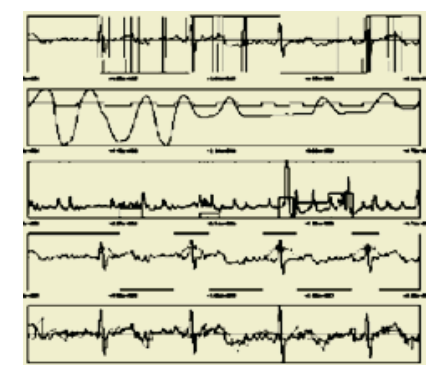

(b)

Fig. 1. A sketch of the extracted features: (a) EGC parameters; (b) coded signals.

The features are compressed into 10 propositional variables through a multilayer perceptron with $80 \%$ of pruned connections. To render the symbols sensitive to a short-term dynamics of the features the input layer is made of 30 input nodes, feed by a normalized version of the ten features at time $t-1 t$, and $t+1$. The output nodes compute a Boolean vector of ten prpositional variables and the hidden layer has ten nodes as well. The number of connections between neurons are 253 in total. The way of training such a network is not trivial, passing through a RAAM architecture and special entropic error function called "edge pulling" elsewhere [1]. We look for a set of vector of Boolean variables where an almost "one to one" correspondence must exist with the feature vectors, but Boolean assignments may coincide when they code data patterns with the same values of the features of interest to us. We succeed in this target apart for around $20 \%$ of examples that met inconsistency, being coded by vectors labeled both by 1 in some examples and by 0 in other. Wether the inconsistency is due to human segmentation or neural classification error is still under investigation.

In order to maintain some understandability of the awareness detection rules, or at least its formal manageability within wider contexts, we required these rules to be symbolic, being expressed on particular as either conjunctive or disjunctive normal forms. We rely on the first level of the PACmeditation procedure discussed in [3] for getting a DNF or a CNF as classifier, and simplify their expressions according to the fuzzy-relaxation procedure discussed in [2]. An example of obtained rule is $x_{4} x_{8} x_{6}+$ $x_{5} x_{3} x_{7} x_{6}+x_{13} x_{10} x_{6}+x_{14} x_{11} x_{6} x_{7} x_{13}+x_{9} x_{7} x_{1} x_{3}+x_{7} x_{13} x_{6} x_{8}+x_{10} x_{3} x_{6} x_{1}+x_{8} x_{1} x_{7} x_{12}+x_{4} x_{8} x_{10} x_{13}$.

\section{Numerical Results and Conclusions}

Figure 2 refers to frames of a clip recording a driving simulation experiment. The lowermost graphs report the driving parameters recorded by the simulator, denoting car acceleration, braking strength, wheel angle, distance from the center line. The 
uppermost graphs show two Boolean variables: dark bars are up when an attention requiring episode occurs according to our above empirical rules; gray bars are up when the system detects a driver attentional state.

The first frame captures the beginning of a foggy tract where the attention of the driver is raised up in spite of absence of particular episodes denoted by the driving parameters. The second one is the crucial instant of a crash due to a "maniac" car suddenly invading the driver's lane. Contemporarily episode and attention bars raise up, but without any chance of avoiding the crash.

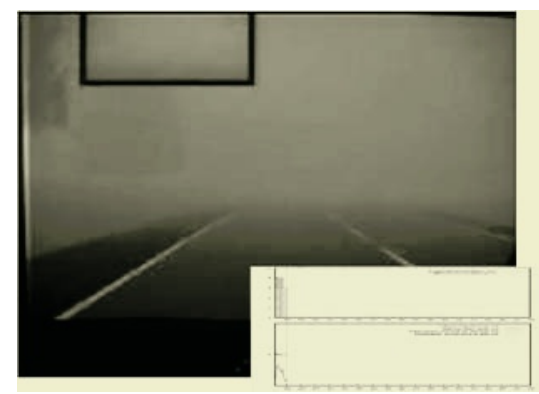

(a)

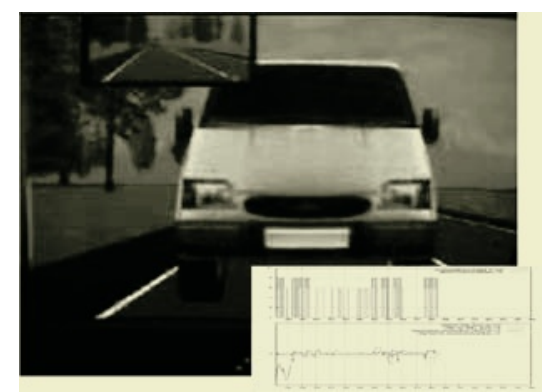

(b)

Fig. 2. Awareness monitoring spots.

The demo will show the entire clip and the companion graphs, denoting how the proposed system detects the awareness or non-awareness state of the driver during various guidance episodes.

\section{References}

1. B. Apolloni, S. Bassis, A. Brega, S. Gaito, D. Malchiodi and A. M. Zanaboni, A manmachine human interface for a special device of the pervasive computing world, in Proceedings of DC Tales, CTI Press.

2. B. Apolloni, D. Malchiodi, C. Orovas and A. M. Zanaboni, Fuzzy methods for simplifying a boolean formula inferred from examples, in Proceedings of FSKD’02, Vol. 2, 554-558.

3. B. Apolloni, F. Baraghini and G. Palmas, Pac meditation on Boolean Formulas, Abstraction, Reformulation and Approximation, Springer, Berlin: 274-281,2002.

4. B. Apolloni, D. Malchiodi, C. Orovas and G. Palmas. From synapses to rules. Cognitive Systems Research, 3(2): 167-201, 2002.

5. R. Plonsey and D. G. Fleming. Bioelectric phenomena. McGraw-Hill, 1969. 